\section{Serous cystadenoma of pancreas: imaging diagnosis}

\section{Case}

A 70-year-old female presented with occasional abdominal pain. There was no jaundice, loss of appetite, loss of weight or fever. She underwent transabdominal ultrasound (US) which showed well circumscribed lobulated echogenic mass in the pancreatic head region with peripheral anechoic cysts and foci of calcification (Figure 1). Multi-phase CT (MPCT) images showed well-defined lobulated hypodense mass composed of multiple cysts (more than 6 in number) of sizes less than $2 \mathrm{~cm}$ with enhancing thin septation and calcified central stellate scar (Figure 2). The mass had a well-defined fat plane with the surrounding structures with no evidence of invasion or compression. There was fatty replacement of body and tail of pancreas with normal caliber main pancreatic duct (MPD).

On MRI (Figure 3), the lesion was lobulated, and welldefined, present in the head of pancreas, composed of multiple, cysts and size less than $2 \mathrm{~cm}$ appearing hypointense on $\mathrm{T} 1 \mathrm{~W}$ and hyperintense on $\mathrm{T} 2 \mathrm{~W}$ images with central area of $\mathrm{T} 1$ and T2 hypointensities suggestive of central stellate scar. The thin

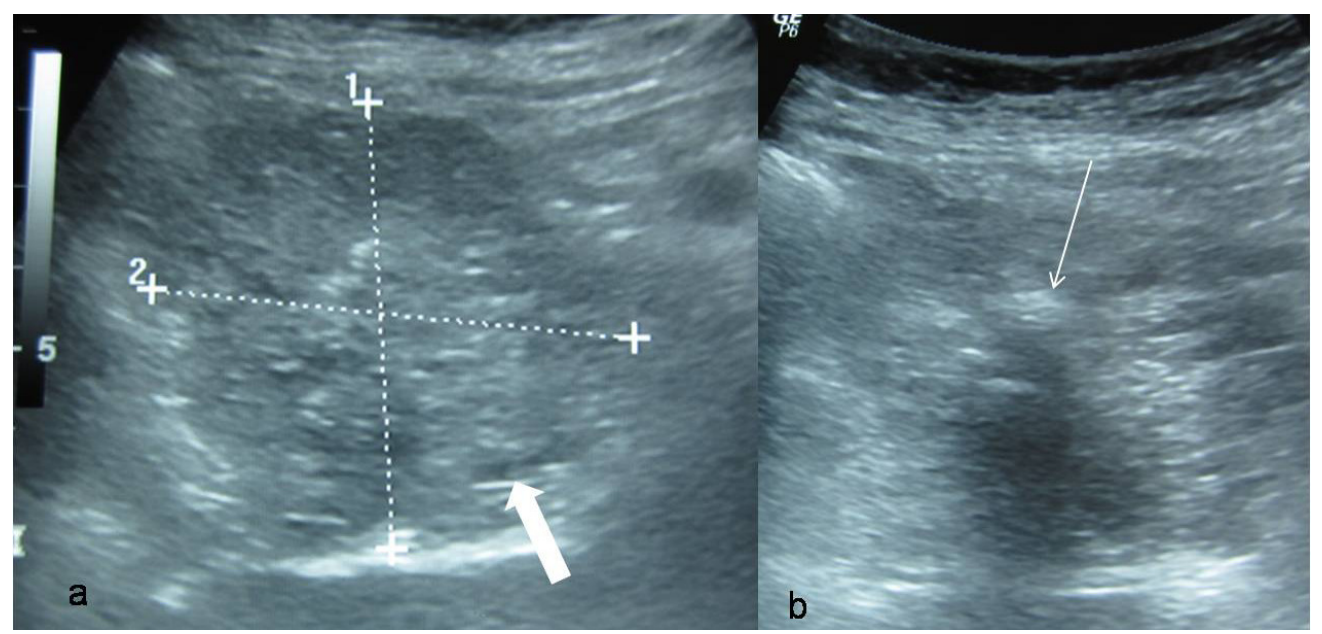

Figure 1: Ultrasound scan $(a, b)$ shows a well defined echogenic mass lesion in pancreatic head region with few anechoic cystic area (thick arrow) in the periphery and calcification (thin arrow) with posterior acoustic shadowing within the mass

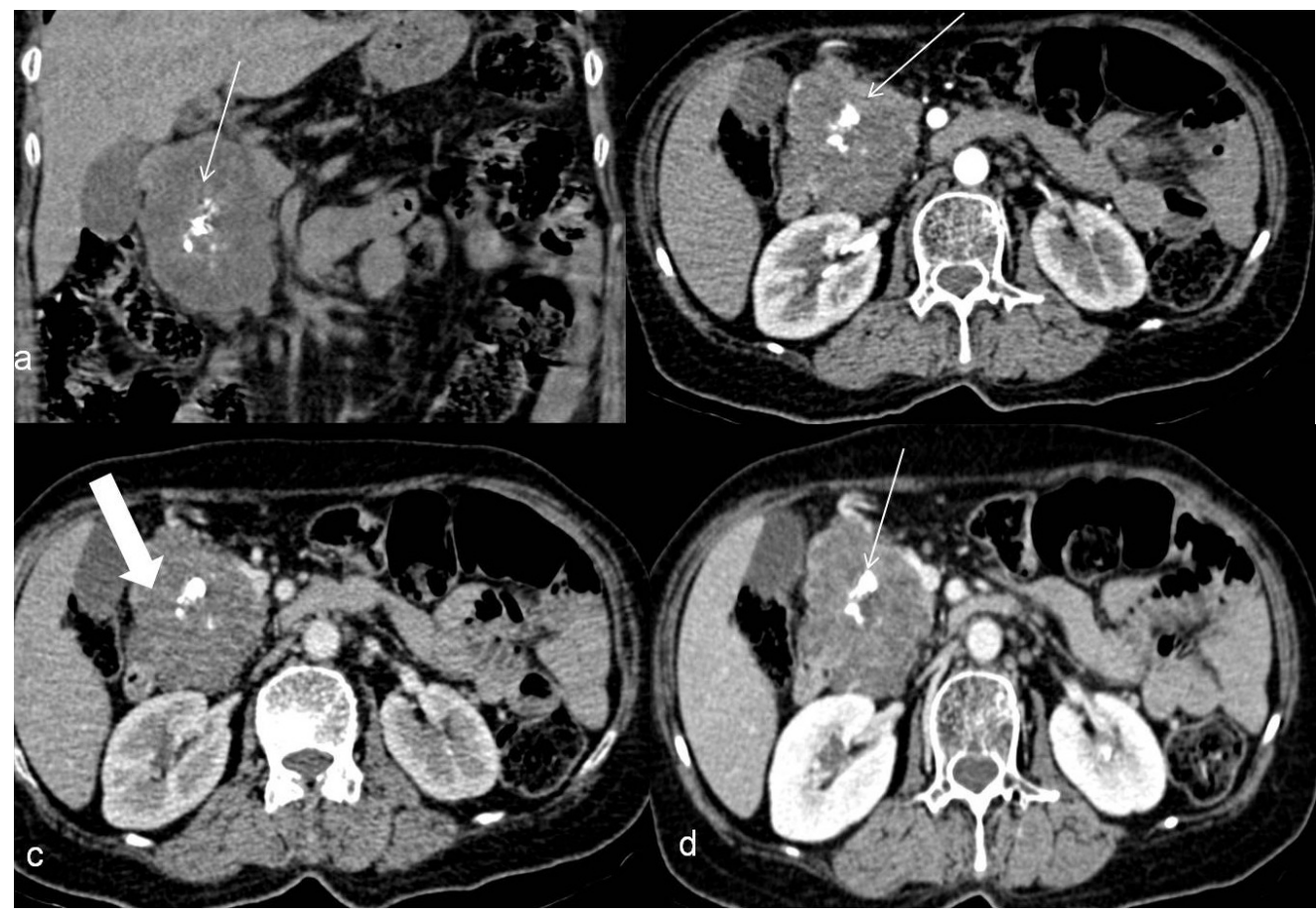

Figure 2: $\quad$ Multiphase contrast enhanced CT (a) NCCT coronal reformat (b) axial section in arterial phase (c) axial section in pancreatic phase (d) axial section in venous phase shows a well defined hypodense lobulated multicystic mass lesion. The periphery of the mass shows relatively nonenhancing area (thick arrow) and the center shows a stellate calcification (thin arrow). 


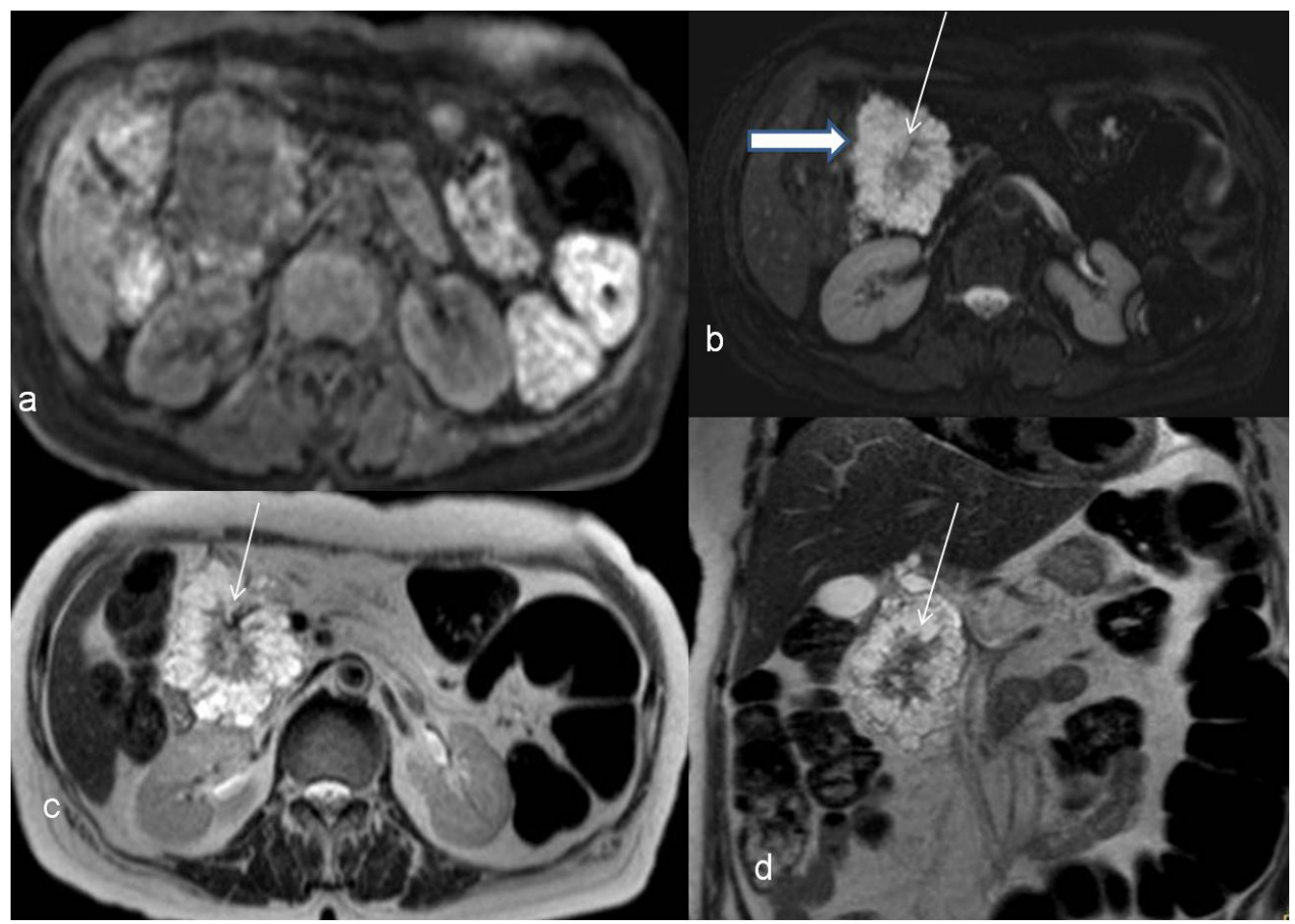

Figure 3: MRI (a) T1 weighted fat suppressed image axial section (b) T2W fat suppressed axial section (c) T2W axial section and (d) T2W coronal section showed well defined lobulated multicystic mass (thick arrow) in head of pancreas.

fibrous septations appeared hypointense on both $\mathrm{T} 1 \mathrm{~W}$ and $\mathrm{T} 2 \mathrm{~W}$ images. There was no evidence of diffusion restriction or signal drop on chemical shift imaging within the mass. No mural nodularity or solid component was seen within the mass. MPD was not seen to communicate with the mass.

Based on the typical radiological features on US, MPCT and MRI, diagnosis of microcystic serous cystadenoma of head of pancreas was made. Complete blood count, liver function test, amylase level, CA 19-9, CEA levels were within normal limits. The patient is on follow up.

\section{Discussion}

Serous cystadenoma of pancreas is a rare benign cystic neoplasm constituting 1-2\% of all exocrine pancreatic tumors and isn't it $10-15 \%$ of all cystic pancreatic neoplasms. ${ }^{1}$ It is usually found incidentally on cross-sectional imaging done for other indications. It is more commonly seen in women (about $80 \%$ ) older than 60 years and has also been referred to as grandmother lesion. They occur most commonly in the head of the pancreas with size range of few millimeters to $20 \mathrm{~cm}$. They have three typical morphological patterns: polycystic, honeycomb and oligocystic. ${ }^{2}$ The polycystic variety accounts for $70 \%$ of total serous cystadenoma consisting of multiple cysts of more than six in number with size less than $2 \mathrm{~cm}$.
Honeycomb pattern accounts for $20 \%$ and oligocystic which is also known as macrocystic variety has cysts of size more than $2 \mathrm{~cm}$ and constitutes about $10 \%$. Central stellate scar with calcification is seen in about $30 \%$ of the patients. ${ }^{3}$ Giant tumors with ductal dilatation, intratumoral hemorrhages, solid variants, unilocular cystic form, interval growth and disseminated forms are atypical presentations of serous cystadenoma. ${ }^{4}$ It is also associated with Von Hippel Lindau disease.

\section{AJAY KUMAR YADAV ${ }^{1}$ DEVASENATHIPATHY KANDASAMY ${ }^{1}$, RAJU SHARMA ${ }^{1}$, PEUSH SAHNI ${ }^{2}$}

Correspondence: Dr. Raju Sharma Deptt. Of Radiodiagnosis ${ }^{l}$ and Deptt. Of GI Surgery and Liver Transplantation All India Institute of medical sciences New Delhi- 110029 Email: raju152@yahoo.com

\section{References}

1. Solicia E, Capella C, Kloppel G. Tumors of theexocrine pancreas. 3rd series, fasc. 20. In: Rosai J,Sorbin L, editors. Atlas of tumor pathology. Washington,DC: Armed Forces Institute of Pathology; 1997.p.31-144.

2. Sarr MG, Murr M, Smyrk TC, Yeo CJ, Fernandez-del-Castillo 
C, Hawes RH,et al. Primary cysticneoplasms of the pancreas: neoplastic disordersof emerging importance — current state-oftheart and unanswered questions. J Gastrointest Surg. 2003;7:417-28.

3. Choi JY, Kim MJ, Lee JY, Lim JS, Chung JJ, Kim KW, et al.Typical and Atypical Manifestations of Serous Cystadenoma of thePancreas: Imaging Findings with Pathologic Correlation. AJR 2009; 193:136-42.

4. Dewhurst CE, Mortele KJ. Cystic Tumors of the Pancreas: Imaging and Management. Radiol Clin N Am. 2012;50:467-86.

\section{Hilar traumatic neuroma masquerading as Klatskin tumor}

\section{Introduction}

Traumatic neuroma is an exuberant but nonneoplastic proliferation of a nerve that occurs after injury or surgery. ${ }^{1,2}$ They have no specific imaging findings and it is very hard to distinguish a traumatic neuroma from a malignant tumor before surgery. After biliary surgery, neuromas can occur in the cystic duct stump but neuromas involving the bile duct that present as hilar tumors are very rare. We present the case of a traumatic neuroma of the bile duct mimicking hilar cholangiocarcinoma.

\section{Case report}

A 69-year-old woman presented with itching of one-year duration. She had jaundice associated with clay colored stools. There was history of loss of weight and appetite. There was no history of fever, abdominal pain, upper GI bleed and no past history suggestive of hepatic decompensation. There was a past history of laparoscopy converted to open cholecystectomy in 2010 after which she developed a large abdominal swelling which gradually increased in size over time. She was moderately built and nourished.. Icterus was present. There was no pallor, cyanosis, clubbing or edema. There was no supraclavicular lymphadenopathy. Pruritus marks were present over her body. Abdominal examination revealed fullness present in right upper abdomen and a right paramedian scar, which was $12 \mathrm{~cm} \times 10 \mathrm{~cm}$ incisional hernia with large defect present around the scar. Vitals were normal. Abnormal laboratory investigations included raised total bilirubin (2.4 $\mathrm{mg} / \mathrm{dL}$ ), raised alkaline phosphatase (596 IU/L), raised GGTP (808 IU/L) and raised alanine transaminase levels (98 IU/L). Serum CA 19.9 was $136.1 \mathrm{U} / \mathrm{ml}$. Hepatitis serology for $\mathrm{HbsAg}$, anti-HCV, HIV1\&II was negative. Autoimmune serology was negative. On evaluation with CECT, there was evidence of prior surgical intervention and post-operative changes seen in the form of prior cholecystectomy with surgical clips seen at the porta hepatic in close vicinity of the biliary confluence. There was an intra-ductal enhancing soft tissue lesion involving the proximal common duct extending upto the primary biliary confluence with bilobar IHBR dilatation and diagnosis of hilar cholangiocarcinoma was rendered (Figure 1). The patient was taken up for surgery after cardiopulmonary evaluation and clearance. Preoperative diagnosis was hilar cholangiocarcinoma type IIIA, post right portal vein embolization and giant incisional hernia. Procedure performed was staging laparotomy with right hepatectomy with caudate lobe resection and CBD resection. Intraoperative distal bile duct resection margin sent for histopathological frozen section examination was negative for dysplasia and malignancy.

Macroscopically, cut surface of the resected specimen showed a greyish-white firm tumour like lesion composed of nodules measuring together $1.2 \mathrm{~cm}$ and grossly involved proximal common bile duct, common hepatic duct \& left hepatic duct (Figure 2). Histological examination revealed hyperplastic and haphazard proliferation of nerve fibres. These nerve fascicles were well-encapsulated and comprised of small bland Schwann cells and tiny axons, endoneurial cells lying in collagenous stroma (Figures 3 and 4) No evidence of malignancy, atypical mitosis or necrosis was seen in the multiple sections obtained after extensive dissection of the resected biliary system. Immunohistochemical analysis showed S-100 staining of the nerve fibres (Figure 5). Sections from the liver

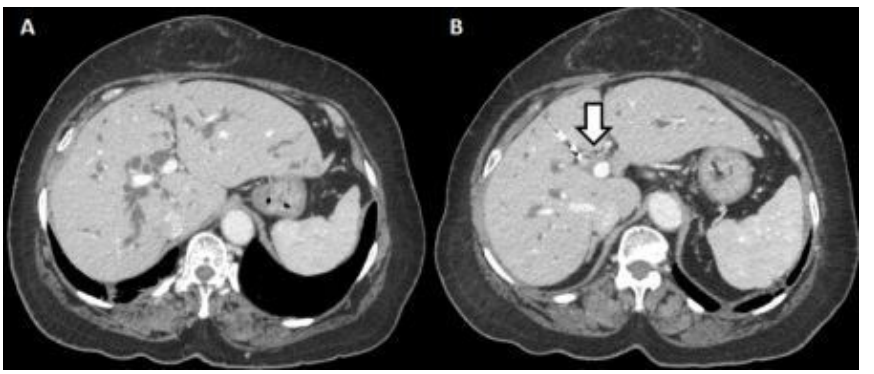

Figure 1: Axial contrast enhanced CT images reveal bilobar intrahepatic biliary dilatation (A) with a subtle ill-defined soft-tissue involving the primary biliary confluence (arrow in B). The lesion is seen in close proximity with the post-cholecystectomy metallic clips. 